\title{
Systematic method for thermomechanically consistent coarse graining: a universal model for methacrylate-based polymers
}

Hsu, David, daviddhsu@gmail.com; Xia, Wenjie; Keten, Sinan, Northwestern University, United States; Arturo, Steven, Dow Chemical Company, United States;

\begin{abstract}
We present a versatile systematic two-bead-per-monomer coarse-grain modeling strategy for simulating the thermomechanical behavior of methacrylate polymers at length and time scales far exceeding atomistic simulations. We establish generic bonded interaction parameters via Boltzmann inversion of probability distributions obtained from the common coarse-grain bead center locations of five different methacrylate polymers. Distinguishing features of each monomer side-chain group are captured using Lennard-Jones nonbonded potentials with parameters specified to match the density and glass-transition temperature values obtained from all-atomistic simulations. The developed force field is validated using Flory-Fox scaling relationships, self-diffusion coefficients of monomers, and modulus of elasticity for $p$ (MMA). Our approach establishes a transferable, efficient, and accurate scalebridging strategy for investigating the thermomechanics of copolymers, polymer blends, and nanocomposites.
\end{abstract}

